KYUNGPOOK Math. J. 49(2009), 57-65

\title{
On Approximation by Post-Widder and Stancu Operators Preserving $x^{2}$
}

LUCYNA REMPULSKA*

Institute of Mathematics, Poznan University of Technology Piotrowo 3A, 60-965

Poznan, Poland

e-mail: lrempuls@math.put.poznan.pl

MARIOLA SKORUPKA

Institute of Mathematics, Poznan University of Technology Piotrowo 3A, 60-965

Poznan, Poland

e-mail: mariolas@math.put.poznan.pl

AbstraCt. In the papers [5]-[7] was examined approximation of functions by the modified Szász-Mrakyan operators and other positive linear operators preserving $e_{2}(x)=x^{2}$. In this paper we introduce the Post-Widder and Stancu operators preserving $x^{2}$ in polynomial weighted spaces. We show that these operators have better approximation properties than classical Post-Widder and Stancu operators.

\section{Introduction}

1.1. The Post-Widder operators

$$
\begin{aligned}
P_{n}(f ; x) \equiv P_{n}(f(t) ; x) & :=\int_{0}^{\infty} f(t) p_{n}(x, t) d t, \quad x \in I, \quad n \in N, \\
p_{n}(x, t) & :=\frac{(n / x)^{n} t^{n-1}}{(n-1) !} \exp \left(-\frac{n t}{x}\right),
\end{aligned}
$$

$I=(0, \infty), N=\{1,2, \cdots\}$, were examined in many papers and monographs (e.g. [4]) for real-valued functions $f$ bounded on $I$. It is known ([4], Chapter 9) that $P_{n}$ are well defined also for functions $e_{k}(x)=x^{k}, k \in N_{0}=N \cup\{0\}$, and

$$
P_{n}\left(e_{0} ; x\right)=1, \quad P_{n}\left(e_{1} ; x\right)=x, \quad P_{n}\left(e_{2} ; x\right)=\frac{n+1}{n} x^{2}
$$

and generally

$$
P_{n}\left(e_{k} ; x\right)=\frac{n(n+1) \cdots(n+k-1) x^{k}}{n^{k}}, \quad k \in N
$$

\footnotetext{
* Corresponding author.
}

Received 19 June 2007; accepted 23 October 2007.

2000 Mathematics Subject Classification: 41A25, 41A36.

Key words and phrases: Post-Widder operator, Stancu operator, polynomial weighted space, approximation theorem. 
for $x \in I$ and $n \in N$. Denoting by

$$
\varphi_{x}(t):=t-x \text { for } t \in I \text { and a fixed } x \in I,
$$

we have

$$
P_{n}\left(\varphi_{x}^{2}(t) ; x\right)=\frac{x^{2}}{n} \quad \text { for } x \in I, n \in N .
$$

From the results given in [4], Chapter 9, we can deduce that for every function $f$ continuous and bounded on $I$ there holds

$$
\left|P_{n}(f ; x)-f(x)\right| \leq M \omega\left(f ; \frac{x}{\sqrt{n}}\right), \quad x \in I, \quad n \in N,
$$

where $\omega(f ; \cdot)$ is the modulus of continuity of $f$ and $M=$ const. $>0$ independent on $x$ and $n$.

1.2. The Stancu operators

$$
\begin{gathered}
L_{n}(f ; x) \equiv L_{n}(f(t) ; x):=\int_{0}^{\infty} f(t) s_{n}(x, t) d t, \quad x \in I, \quad n \in N, \\
s_{n}(x, t):=\frac{1}{B(n x, n+1)} \frac{t^{n x-1}}{(1+t)^{n x+n+1}},
\end{gathered}
$$

with the Euler beta function

$$
B(a, b):=\int_{0}^{1} t^{a-1}(1-t)^{b-1} d t \equiv \int_{0}^{\infty} \frac{t^{a-1}}{(1+t)^{a+b}} d t, \quad a, b>0,
$$

were introduced in [10] for real-valued functions $f$ bounded and locally integrable on $I=(0, \infty)$. The Stancu operators $L_{n}$ are also well defined for functions $e_{k}(x)=x^{k}$, $k \in N_{0}$, (see [10], [1], [2]) and

$$
\begin{aligned}
& L_{n}\left(e_{0} ; x\right)=1, \quad L_{n}\left(e_{1} ; x\right)=x, \quad \text { for } n \in N, \\
& L_{n}\left(e_{2} ; x\right)=x^{2}+\frac{x(x+1)}{n-1} \quad \text { for } n \geq 2,
\end{aligned}
$$

and generally

$$
L_{n}\left(e_{k} ; x\right)=\frac{n x(n x+1) \cdots(n x+k-1)}{n(n-1) \cdots(n-k+1)}, \quad x \in I, \quad n \geq k \geq 2 .
$$

In [10] was proved that for every function $f$ continuous and bounded on $I$ there holds the following inequality

$$
\left|L_{n}(f ; x)-f(x)\right| \leq(1+\sqrt{x(x+1)}) \omega\left(f ; \frac{1}{\sqrt{n-1}}\right)
$$


for $x \in I$ and $n \geq 2$, where $\omega(f ; \cdot)$ is the modulus of continuity of $f$.

1.3. In papers [8] and [9] were examined approximation properties certain modified Post-Widder and Stancu operators for differentiable functions in polynomial weighted spaces. In [5] were investigated modified Szász-Mirakyan operators $D_{n}^{*}$ preserving the function $e_{2}(x)=x^{2}$ and was proved that these operators have better approximation properties than classical Szász-Mirakyan operators. The similar results were given for certain positive linear operators in the papers [6] and [7].

1.4. The purpose of this note is to investigate modified Post-Widder and Stancu operators $P_{n}^{*}$ and $L_{n}^{*}$ preserving $e_{2}(x)=x^{2}$ in polynomial weighted spaces. These operators have better approximation properties than $P_{n}$ and $L_{n}$ given by (1) and (8). The definition and some properties of operators $P_{n}^{*}$ and $L_{n}^{*}$ will be given in Section 2. The main theorems will be given in Section 3 .

1.5. First we give definition of polynomial weighted space $C_{r}$.

Similarly to [3] let $r \in N_{0}$,

$$
w_{0}(x):=1, \quad w_{r}(x):=\left(1+x^{r}\right)^{-1} \quad \text { if } \quad r \geq 1, \quad x \in I,
$$

and let $C_{r} \equiv C_{r}(I)$ be the set of all real-valued functions $f$ defined on $I$, for which $w_{r} f$ is uniformly continuous and bounded on $I$ and the norm is given by

$$
\|f\|_{r} \equiv\|f(\cdot)\|_{r}:=\sup _{x \in I} w_{r}(x)|f(x)| .
$$

It is obvious that if $q<r$, then $C_{q} \subset C_{r}$ and $\|f\|_{r} \leq\|f\|_{q}$ for $f \in C_{q}$. For $f \in C_{r}$, $r \in N_{0}$, we shall consider the modulus of continuity

$$
\omega\left(f ; C_{r} ; t\right):=\sup _{0 \leq h \leq t}\left\|\Delta_{h} f(\cdot)\right\|_{r}, \quad t \geq 0,
$$

where $\Delta_{h} f(x)=f(x+h)-f(x)$.

In this paper we shall apply the following inequalities

$$
\left(w_{r}(x)\right)^{2} \leq w_{2 r}(x), \quad\left(w_{r}(x)\right)^{-2} \leq 4\left(w_{2 r}(x)\right)^{-1},
$$

for $x \in I$ and $r \in N_{0}$, which immediately result from (13).

We shall denote by $M_{i}(r), i \in N$, suitable positive constants depending only on indicated parameter $r$.

\section{The definition and elementary properties of $P_{n}^{*}$ and $L_{n}^{*}$}

2.1. We introduce for $f \in C_{r}, r \in N_{0}$, the following modified Post-Widder operators $P_{n}^{*}$

$$
P_{n}^{*}(f ; x):=\int_{0}^{\infty} f(t) p_{n}\left(u_{n}(x), t\right) d t=P_{n}\left(f ; u_{n}(x)\right), \quad x \in I, \quad n \in N
$$


where $P_{n}(f)$ and $p_{n}$ are given by (1) and (2) and

$$
u_{n}(x):=\sqrt{\frac{n}{n+1}} x,
$$

and modified Stancu operators

$$
L_{n}^{*}(f ; x):=\int_{0}^{\infty} f(t) s_{n}\left(v_{n}(x), t\right) d t=L_{n}\left(f ; v_{n}(x)\right)
$$

for $x \in I$ and $n \geq r \geq 2$ or $n \geq 2$ if $r=0,1$, where $L_{n}(f)$ and $s_{n}$ are given by (8) and (9) and

$$
v_{n}(x):=\frac{-1+\sqrt{1+4 n(n-1) x^{2}}}{2 n} .
$$

2.2. The formulas (18) and (20) imply that

$$
0<u_{n}(x)<x, \quad 0 \leq v_{n}(x) \leq x \quad \text { for } x \in I, \quad n \in N
$$

From (17)-(20) and (1)-(4) and (8)-(11) we immediately obtain the following

Lemma 1. Let $e_{k}(x)=x^{k}$ for $k \in N_{0}$ and $x \in I$. Then for all $x \in I$ and $n \in N$ we have

$$
P_{n}^{*}\left(e_{0} ; x\right)=1, \quad P_{n}^{*}\left(e_{1} ; x\right)=u_{n}(x), \quad P_{n}^{*}\left(e_{2} ; x\right)=x^{2}
$$

and

$$
P_{n}^{*}\left(e_{k} ; x\right)=\frac{n(n+1) \cdots(n+k-1) u_{n}^{k}(x)}{n^{k}} \quad \text { if } k \geq 3 .
$$

Moreover, for $x \in I$ and $n \geq 2$ we have

$$
L_{n}^{*}\left(e_{0} ; x\right)=1, \quad L_{n}^{*}\left(e_{1} ; x\right)=v_{n}(x), \quad L_{n}^{*}\left(e_{2} ; x\right)=x^{2}
$$

and generally

$$
L_{n}^{*}\left(e_{k} ; x\right)=\frac{n v_{n}(x)\left(n v_{n}(x)+1\right) \cdots\left(n v_{n}(x)+k-1\right)}{n(n-1) \cdots(n-k+1)} \text { for } n \geq k \geq 2 .
$$

The formulas (22) and (23) show that $P_{n}^{*}$ and $L_{n}^{*}$ preserve the functions $e_{0}$ and $e_{2}$

Lemma 2. For function $\varphi_{x}$ given by (5) there hold the following analogies of (6):

$$
P_{n}^{*}\left(\varphi_{x}^{2}(t) ; x\right)=2 x\left(x-u_{n}(x)\right) \leq \frac{x^{2}}{n} \quad \text { for } x \in I, \quad n \in N,
$$

and

$$
L_{n}^{*}\left(\varphi_{x}^{2}(t) ; x\right)=2 x\left(x-v_{n}(x)\right) \leq \frac{x(x+1)}{n-1} \text { for } x \in I, \quad n \geq 2 .
$$


Proof. We shall prove only (25) because the proof of (24) is analogous. By linearity of $L_{n}^{*}$ and (5) and (23) we have

$$
\begin{aligned}
L_{n}^{*}\left(\varphi_{x}^{2}(t) ; x\right) & =L_{n}^{*}\left(e_{2} ; x\right)-2 x L_{n}^{*}\left(e_{1} ; x\right)+x^{2} L_{n}^{*}\left(e_{0} ; x\right) \\
& =2 x\left(x-v_{n}(x)\right) \quad \text { for } x>0, \quad n \geq 2 .
\end{aligned}
$$

Next, by (20) we get

$$
\begin{aligned}
0<x-v_{n}(x) & =\frac{2 n x+1-\sqrt{1+4 n(n-1) x^{2}}}{2 n} \\
& =\frac{2 x(x+1)}{2 n x+1+\sqrt{1+4 n(n-1) x^{2}}} \leq \frac{2 x(x+1)}{2 n x+1+2(n-1) x} \\
& \leq \frac{2 x(x+1)}{4(n-1) x}=\frac{x+1}{2(n-1)} \quad \text { for } x>0, n \geq 2 .
\end{aligned}
$$

This completes the proof of (25).

Lemma 3. Let $r \in N_{0}$ and let $w_{r}$ be the weighted function given by (13). Then for $n \in N$ the following inequalities

$$
\begin{gathered}
\left\|P_{n}^{*}\left(1 / w_{r}\right)\right\|_{r} \leq 1, \quad\left\|L_{n}^{*}\left(1 / w_{r}\right)\right\|_{r} \leq 1 \quad \text { if } r=0,1, \\
\left\|P_{n}^{*}\left(1 / w_{r}\right)\right\|_{r} \leq 1+(r-1) !, \quad \text { if } r \geq 2,
\end{gathered}
$$

and

$$
\left\|L_{n}^{*}\left(1 / w_{r}\right)\right\|_{r} \leq 1+2^{2 r-1}\left(1+r^{r-1}\right) \quad \text { for } n \geq r \geq 2
$$

hold. Moreover, for every $f \in C_{r}$ we have

$$
\begin{gathered}
\left\|P_{n}^{*}(f)\right\|_{r} \leq\|f\|_{r}\left\|P_{n}^{*}\left(1 / w_{r}\right)\right\|_{r}, \quad n \in N, \\
\left\|L_{n}^{*}(f)\right\|_{r} \leq\|f\|_{r}\left\|L_{n}^{*}\left(1 / w_{r}\right)\right\|_{r}, \quad n \geq r .
\end{gathered}
$$

The formulas (17)-(20) and inequalities (29) and (30) show that $P_{n}^{*}, n \in N$, and $L_{n}^{*}$ with $n \geq r$ are positive linear operators acting from the space $C_{r}$ to $C_{r}, r \in N_{0}$.

Proof. Similarly to Lemma 2 we shall consider only operators $L_{n}^{*}$. The inequality (26) is obvious by (13), (23), (21) and (14). If $r \geq 2$, then by linearity of $L_{n}^{*}$ and 
(13), Lemma 1 and (21) we get

$$
\begin{aligned}
L_{n}^{*}\left(1 / w_{r} ; x\right) & =L_{n}^{*}\left(e_{0} ; x\right)+L_{n}^{*}\left(e_{r} ; x\right) \\
& \leq 1+\frac{n x(n x+1) \cdots(n x+r-1)}{n(n-1) \cdots(n-r+1)} \\
& \leq 1+\frac{n^{r-1} x(x+1 / n) \cdots(x+(r-1) / n)}{(n-1)(n-2) \cdots(n-r+1)} \\
& \leq 1+\frac{2^{r-1}\left\{(n-r+1)^{r-1}+r^{r-1}\right\}(x+1)^{r}}{(n-r+1)^{r-1}} \\
& \leq 1+2^{2 r-1}\left(1+r^{r-1}\right)\left(1+x^{r}\right)
\end{aligned}
$$

for $x \in I$ and $n \geq r$. This inequality and (14) imply (28).

The inequality (30) immediately follows from (19) and (14).

Applying the Hölder inequality and Lemma 2, Lemma 3 and (16), we easily obtain the following

Lemma 4. Let $r \in N_{0}$ and let $\varphi_{x}$ be given by (5). Then there exist $M_{i}(r)=$ const. $>0, i=1,2$, such that for $x \in I$ and $n \in N$

$$
w_{r}(x) P_{n}^{*}\left(\frac{\left|\varphi_{x}(t)\right|}{w_{r}(t)} ; x\right) \leq M_{1}(r) \sqrt{2 x\left(x-u_{n}(x)\right)}
$$

and

$$
w_{r}(x) L_{n}^{*}\left(\frac{\left|\varphi_{x}(t)\right|}{w_{r}(t)} ; x\right) \leq M_{2}(r) \sqrt{2 x\left(x-v_{n}(x)\right)}, \text { for } n \geq 2 r
$$

\section{Theorems}

3.1. Denote by $C_{r}^{1} \equiv C_{r}^{1}(I)$, with a fixed $r \in N_{0}$, the set of all functions $f \in C_{r}$ which the first derivative belonging also to $C_{r}$.

Theorem 1. Let $r \in N_{0}$. Then there exist $M_{i}(r)=$ const. $>0, i=3,4$, such that for every $f \in C_{r}^{1}, x \in I$ and $n \in N$ the following inequalities

$$
w_{r}(x)\left|P_{n}^{*}(f ; x)-f(x)\right| \leq M_{3}(r)\left\|f^{\prime}\right\|_{r} \sqrt{2 x\left(x-u_{n}(x)\right)}
$$

and

$$
w_{r}(x)\left|L_{n}^{*}(f ; x)-f(x)\right| \leq M_{4}(r)\left\|f^{\prime}\right\|_{r} \sqrt{2 x\left(x-v_{n}(x)\right)}, \quad n \geq 2 r,
$$

hold.

Proof. From (17), (18) and Lemma 1 we deduce that

$$
\left|P_{n}^{*}(f(t) ; x)-f(x)\right|=\left|P_{n}^{*}(f(t)-f(x) ; x)\right| \leq P_{n}^{*}\left(\left|\int_{x}^{t} f^{\prime}(y) d y\right| ; x\right)
$$


for every $f \in C_{r}^{1}, x \in I$ and $n \in N$. Next by (13) and (14) we have

$$
\begin{aligned}
\left|\int_{x}^{t} f^{\prime}(y) d y\right| & \leq\left\|f^{\prime}\right\|_{r}\left|\int_{x}^{t} \frac{d y}{w_{r}(y)}\right| \\
& \leq\left\|f^{\prime}\right\|_{r}\left(\frac{1}{w_{r}(t)}+\frac{1}{w_{r}(x)}\right)|t-x|, \quad x, t \in I .
\end{aligned}
$$

Consequently, we get

$$
w_{r}(x)\left|P_{n}^{*}(f(t) ; x)-f(x)\right| \leq\left\|f^{\prime}\right\|_{r}\left\{P_{n}^{*}\left(\frac{\left|\varphi_{x}(t)\right|}{w_{r}(t)} ; x\right)+P_{n}^{*}\left(\frac{\left|\varphi_{x}(t)\right|}{w_{0}(t)} ; x\right)\right\},
$$

for $x \in I, n \in N$, where $\varphi_{x}$ is defined by (5). Now using (31), we obtain the desired estimation (33).

Similarly, applying (32), we obtain (34).

Theorem 2. Let $r \in N_{0}$. Then there exist $M_{i}(r)=$ const. $>0, i=5,6$, such that for every $f \in C_{r}, x \in I$ and $n \in N$ we have

$$
w_{r}(x)\left|P_{n}^{*}(f ; x)-f(x)\right| \leq M_{5}(r) \omega\left(f ; C_{r} ; \sqrt{2 x\left(x-u_{n}(x)\right.}\right)
$$

and

$$
w_{r}(x)\left|L_{n}^{*}(f ; x)-f(x)\right| \leq M_{6}(r) \omega\left(f ; C_{r} ; \sqrt{2 x\left(x-v_{n}(x)\right.}\right), \quad n \geq 2 r,
$$

where $\omega\left(f ; C_{r}\right)$ is the modulus of continuity of $f$ defined by $(15)$.

Proof. Because the proofs of (35) and (36) are analogous, we shall prove only (35). We shall use the Stieklov function $f_{h}$ of $f \in C_{r}$, i.e.

$$
f_{h}(x):=\frac{1}{h} \int_{0}^{h} f(x+t) d t, \quad x, h>0 .
$$

From (37) and (15) it follows that

$$
\begin{gathered}
\left\|f_{h}-f\right\|_{r} \leq \omega\left(f ; C_{r} ; h\right), \\
\left\|f_{h}^{\prime}\right\|_{r} \leq h^{-1} \omega\left(f ; C_{r} ; h\right),
\end{gathered}
$$

for every $f \in C_{r}$ and $h>0$. These inequalities show that if $f \in C_{r}$ with a fixed $r \in N_{0}$, then $f_{h} \in C_{r}^{1}$ for every $h>0$. Hence for $f \in C_{r}$ and $h>0$ we can write

$$
\begin{aligned}
P_{n}^{*}(f(t) ; x)-f(x)= & P_{n}^{*}\left(f(t)-f_{h}(t) ; x\right)+P_{n}^{*}\left(f_{h}(t) ; x\right)-f_{h}(x) \\
& +f_{h}(x)-f(x) \quad \text { for } x \in I, \quad n \in N .
\end{aligned}
$$

By (29), (26), (27) and (38)we see that there exists $M_{7}(r)=$ constant $>0$ such that

$$
\begin{aligned}
w_{r}(x)\left|P_{n}^{*}\left(f(t)-f_{h}(t) ; x\right)\right| & \leq M_{7}(r)\left\|f-f_{h}\right\|_{r} \\
& \leq M_{7}(r) \omega\left(f ; C_{r} ; h\right) .
\end{aligned}
$$


Applying Theorem 1 for $f_{h}$ and (39), we get

$$
\begin{aligned}
w_{r}(x)\left|P_{n}^{*}\left(f_{h}(t) ; x\right)-f_{h}(x)\right| & \leq M_{3}(r)\left\|f_{h}^{\prime}\right\|_{r} \sqrt{2 x\left(x-u_{n}(x)\right)} \\
& \left.\leq M_{3}(r) h^{-1} \omega\left(f ; C_{r} ; h\right)\right) \sqrt{2 x\left(x-u_{n}(x)\right.}
\end{aligned}
$$

Using (41), (42) and (38), we deduce from (40)

(43) $w_{r}(x)\left|P_{n}^{*}(f ; x)-f(x)\right| \leq M_{8}(r) \omega\left(f ; C_{r} ; h\right) \times\left\{1+h^{-1} \sqrt{2 x\left(x-u_{n}(x)\right)}\right\}$

for $x>0, h>0$ and $n \in N$. Now, for given $x$ and $n$ setting $h=\sqrt{2 x\left(x-u_{n}(x)\right)}$ to (43), we obtain desired inequality (35) and we complete the proof.

From Theorem 2 and Lemma 2 results the following

Corollary. For every $f \in C_{r}, r \in N_{0}$, we have $\lim _{n \rightarrow \infty} P_{n}^{*}(f ; x)=f(x), x \in I$, and this convergence is uniform on every interval $[a, b], a>0$.

The above statement is also true for Stancu operators $L_{n}^{*}$.

3.2. Considering the Stancu operators $L_{n}$ in polynomial weighted spaces $C_{r}$ and using methods of proofs of Theorem 1 and Theorem 2, we can obtain the following estimation

$$
w_{r}(x)\left|L_{n}(f ; x)-f(x)\right| \leq M_{9}(r) \omega\left(f ; C_{r} ; \sqrt{\frac{x(x+1)}{n-1}}\right),
$$

for every $f \in C_{r}, r \in N_{0}, x>0$ and $n \geq 2 r+2$.

The inequalities (44), (36) and (12) show that the Stancu operators $L_{n}^{*}$ have better approximation properties than $L_{n}$ for functions $f \in C_{r}, r \in N_{0}$, and $n \geq$ $2 r+2$. Moreover, by (20) and Lemma 2 we get for arguments of moduli of continuity of $f$ given in (36) and (44)

$$
\begin{aligned}
& \sqrt{\frac{x(x+1)}{n-1}}-\sqrt{2 x\left(x-v_{n}(x)\right)} \\
= & \sqrt{\frac{x(x+1)}{n-1}}-\frac{\sqrt{4 x^{2}(x+1)}}{\sqrt{2 n x+1+\sqrt{1+4 n(n-1) x^{2}}}} \\
= & \sqrt{\frac{x(x+1)}{n-1}}\left(1-\frac{\sqrt{4(n-1) x}}{\sqrt{2 n x+1+\sqrt{1+4 n(n-1) x^{2}}}}\right)
\end{aligned}
$$




$$
\begin{aligned}
& =\sqrt{\frac{x(x+1)}{\sqrt{n-1}}} \frac{\sqrt{1+4 n(n-1) x^{2}}-2(n-1) x+2 x+1}{\sqrt{2 n x+1+\sqrt{1+4 n(n-1) x^{2}}}} \\
& \times \frac{1}{\left[\sqrt{2 n x+1+\sqrt{1+4 n(n-1) x^{2}}}+\sqrt{4(n-1) x}\right]} \\
& >\sqrt{\frac{x(x+1)}{n-1}} \frac{2 x+1}{\sqrt{4 n x+2}[\sqrt{4 n x+2}+\sqrt{4(n-1) x}]} \\
& >\sqrt{\frac{x(x+1)}{n-1}} \frac{2 x+1}{2(4 n x+2)}>\sqrt{\frac{x(x+1)}{n-1}} \frac{1}{4 n},
\end{aligned}
$$

for all $x>0$ and $n \geq 2 r+2$.

Analogously, estimations (7), (35) and (24) show that $P_{n}^{*}, n \in N$, have better approximation properties than $P_{n}$ for functions $f \in C_{r}$ (see [8]). Moreover, by (7), (35) and (18) we can obtain

$$
\frac{x}{\sqrt{n}}-\sqrt{2 x\left(x-u_{n}(x)\right)} \geq \frac{x}{4(n+1) \sqrt{n}} \text { for } x>0 \text { and } n \in N \text {. }
$$

\section{References}

[1] Abel U., Asymptotic approximation with Stancu beta operators,Rev. Anal. Numér. Théor. Approx., 27(1)(1998), 5-13.

[2] Abel U., Gupta V., Rate of convergence for Stancu beta operators for functions of bounded variation, Rev. Anal. Numér. Théor. Approx., 33(1)(2004), 3-9.

[3] Becker M., Global approximation theorems for Szász-Mirakyan and Baskakov operators in polynomial weight spaces, Indiana Univ. Math. J., 27(1)(1978), 127-142.

[4] Ditzian Z., Totik V., Moduli of Smoothness, Springer-Verlag, New York, 1987.

[5] Duman O., Özarslan M. A., Szász-Mirakjan type operators providing a better error estimation, Applied Math. Letters, (in print).

[6] Duman O., Özarslan M. A., MKZ type operators providing a better estimation on [1/2, 1), Cand. Math. Bull., 50(2007), 434-439.

[7] King J. P., Positive linear operators which preservge $x^{2}$, Acta Math. Hungar., 99(2003), 203-208.

[8] Rempulska L., Skorupka M., On strong approximation applied to Post-Widder operators, Anal. in Theory and Applic., 22(2)(2006), 172-182.

[9] Rempulska L., Skorupka M., Approximation properties of modified Stancu beta operators, Rev. Anal. Numér. Théor. Approx., 35(2)(2006), (in print).

[10] Stancu D. D., On the beta approximating operators of second kind, Rev. Anal. Numer. Theor. Approx., 24(1-2)(1995), 231-239. 